\title{
SIMULACIÓN DE LA PROTESTA SOCIOPOLÍTICA
}

\section{Oier Gil Zapirain}

Universidad del País Vasco / Euskal Herriko Unibertsitatea, Dpto. Arte y Tecnología. Doctorando

\section{Resumen}

Este artículo plantea un análisis de Protest-\$1 como proyecto artístico-tecnológico multiusuario, interactivo y relacional que permite al usuario un acercamiento cognitivo a la protesta sociopolítica, siendo parte de una manifestación simulada en contra de los bombardeos de Israel sobre Palestina. La instalación se compone de una proyección frontal, dos altavoces y tres dispositivos compuestos de un megáfono y un smartphone, cada uno. El interactor elige las acciones que desea hacer acontecer en el evento de protesta pulsando los botones de la pantalla táctil del teléfono móvil y posteriormente, agita el megáfono con el fin de materializar en la manifestación, mediante el esfuerzo, la acción escogida. A su vez, Protesta-\$1 permite a los usuarios luchar para evitar que otro usuario consiga sacar adelante la acción de protesta elegida creando un marco en el que el querer de la mayoría somete siempre a la minoría. Los altavoces emiten la voz de los medios de comunicación que relatan la protesta en forma de noticiario y a tiempo real, permitiendo a los interactores articular la protesta siendo conscientes de la imagen global que se está emitiendo.

\section{Palabras-clave: INTERACCIÓN MULTIUSUARIO; ARTICULACIÓN; PROTESTA SOCIOPOLIITICA; MEDIOS DE COMUNICACIÓN; CONECTIVIDAD}

\section{SIMULATING SOCIO-POLITICAL PROTEST}

\section{Abstract}

This paper presents an analysis of Protest-\$1: an artistic-technological, multi-user, interactive, relational project that provides the user with a cognitive approach to the socio-political protest by being part of a simulated demonstration against the Israeli bombing of Palestine. The installation comprises a front projection screen, two loudspeakers and three devices each consisting of a megaphone and a smartphone. The person who is interacting selects the actions that he/she wants to make happen during the protest event by pressing the buttons on the touch screen of a mobile phone and then shaking a megaphone so that, through this effort, the chosen action can be implemented in the demonstration. Likewise, by creating a framework in which the wishes of the majority always prevail over those of the minority, Protest- $\$ 1$ allows the users to fight and thus prevent another user from managing to implement their own chosen protest action. The loudspeakers broadcast the voice of the mass media reporting on the protest in the form of a news bulletin and in real time, thus allowing those interacting to articulate the protest while remaining aware of the global image that is being broadcast.

Keywords: INTERACTION; MULTI-USER; ARTICULATION; SOCIO-POLITICAL PROTEST; MASS MEDIA; CONNECTIVITY

\footnotetext{
Gil Zapirain, 0ier. 2016. "Simulación de la protesta sociopolítica". AusArt 4(1): pp-pp. 175-185 DOI: 10.1387/ausart.16698
}

\section{AUSART}




\section{INTRODUCCIÓN}

La preparación de una estrategia para un evento de protesta sociopolítica es clave para la obtención de los objetivos para los que se protesta. Tal y como escribió Sun Tzu en su Arte de la Guerra (2013), el general que gana la batalla hace muchos cálculos antes de pelear y, en consecuencia, los convocantes de los eventos de queja, construyen un plan de acción orientado a la consecución de dichos objetivos. En el siglo XXI el poder de la información que poseen los medios de comunicación dominantes es tal, que cualquier evento que no sale en estos medios, no existe (Puente 2013). Por ello, es vital que la estrategia de este tipo de eventos tenga en cuenta la imagen e ideas que quiere que sean transmitidas a través de estos medios del poder y recalcar a la multitud movilizada la importancia de la obediencia a lo pactado para conseguir difundir el mensaje tal y como ha sido pre-diseñado.

Sin embargo, son muchas las ocasiones en que un grupo minoritario decide no acatar lo acordado y provocan que todo el evento sufra las consecuencias de sus actos. Un claro ejemplo de este tipo de sucesos es el que padeció la Marcha de la Dignidad que se llevó a cabo el 22 de marzo del 2014 en Madrid. Un pequeño grupo se vio envuelto en disturbios con la policía, propiciando que una manifestación de dos millones de personas que transcurrió pacíficamente ${ }^{1}$ fuese desacreditada en su conjunto. El periódico El Mundo publicó las siguientes líneas: "Al menos ciento un heridos, sesenta y siete de ellos policías y veintinueve detenidos en disturbios tras la 'marcha de la dignidad'" (Durán 2014).

En este tipo de situaciones es frustrante ver que la voluntad de la mayoría no sirve para disuadir a la minoría. Ante esto, se ve la necesidad de crear un marco donde la mayoría no se vea sometida por la minoría y pueda luchar por evitar acciones minoritarias. Así nace Protest-\$1.

\section{DESCRIPCIÓN}

Protest- $\$ 1$ es un proyecto multi-usuario, interactivo y relacional que permite al usuario ser parte de una simulación de una manifestación de protesta sociopolítica en contra del conflicto bélico Palestino-israelí. La instalación se compone de una proyección frontal, dos altavoces que emiten el contenido de los 
medios de comunicación en forma de noticiario y tres dispositivos compuestos de un smartphone adjunto a un megáfono, cada uno.

\subsection{EXPERIENCIA DE USUARIO}

Los usuarios, al adentrarse en el espacio de la instalación, encuentran en el suelo tres megáfonos con un teléfono móvil unido al altavoz mediante un cable (en adelante, cada uno de estos megáfonos-con-móvil será llamado MM y numerado a razón de MM1, MM2 y MM3). Frente a los MM, una proyección muestra el muro de la franja de Gaza tras el cual se visualiza el cielo Palestino coloreado por los bombardeos que suceden tras el muro. El sonido de las bombas acompañan a una voz-en-off que relata el origen del conflicto desde su inicio hasta la actualidad.

Cuando al menos un MM es levantado del suelo por un usuario, la voz-en-off calla, el muro de Gaza desaparece y el usuario se enfrenta a las imágenes de una manifestación pro-palestina convirtiéndose en participante del evento de protesta. En ese momento, en las pantallas de los smartphones de los MM que hasta ese momento permanecían inactivas, se habilitan diversos botones con opciones de protesta que los usuarios pueden elegir. Para hacer acontecer una elección en la manifestación, hay que pulsar en la pantalla táctil el botón elegido y luchar agitando el altavoz. Cuanto más se agite cada MM, más probable será que lo elegido suceda en el evento (en adelante, el proceso de elegir una opción en el MM y luchar por sacarlo adelante será denominado 'duelo').
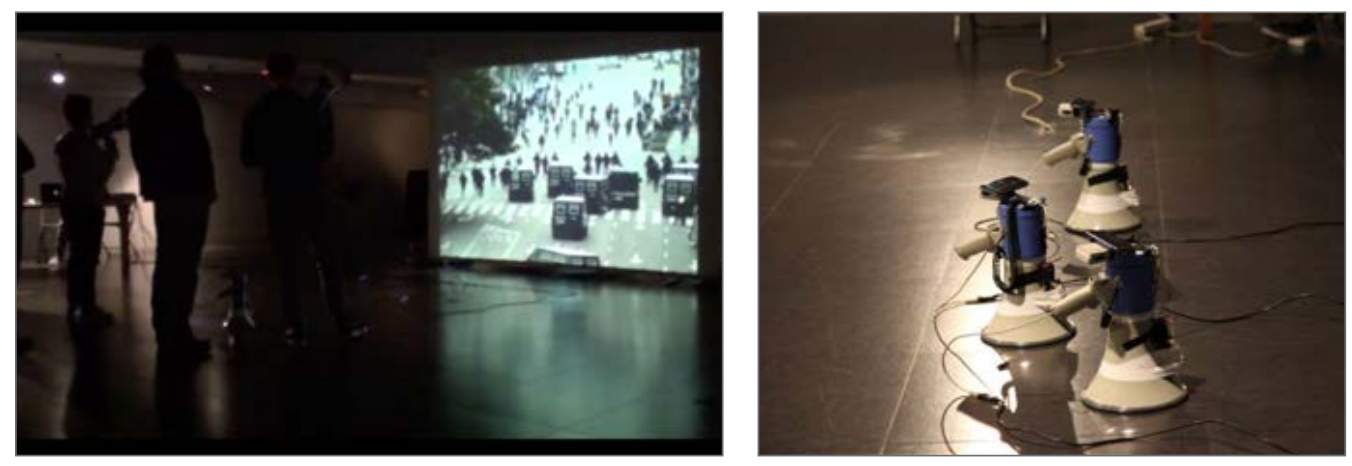

Transversalmente, durante toda la simulación, el apartado sonoro es ocupado por dos tipos de noticias diferentes de los medios de comunicación: noticias objetivas que informan del evento y noticias destructivas que lo desacreditan. 
Las acciones que salen adelante gracias a los duelos de los MM, suben o bajan el volumen de cada tipo de noticias. Las acciones que implican una intervención policial suben el volumen de las noticias destructivas y bajan el de las objetivas. Cada lema elegido, como representación de la protesta por la vía pacífica, sube el volumen de las noticias objetivas y baja el de las destructivas. Cuanto más tiempo transcurre desde el inicio de la protesta, más cantidad de noticias se suman a cada tipo de noticia. Así, el entorno sonoro emite a los usuarios la simulación a tiempo real de la imagen que los medios de comunicación dan al evento de protesta. A cada segundo, los usuarios saben qué es lo que los medios de comunicación transmiten, según las acciones que se han sacado adelante.
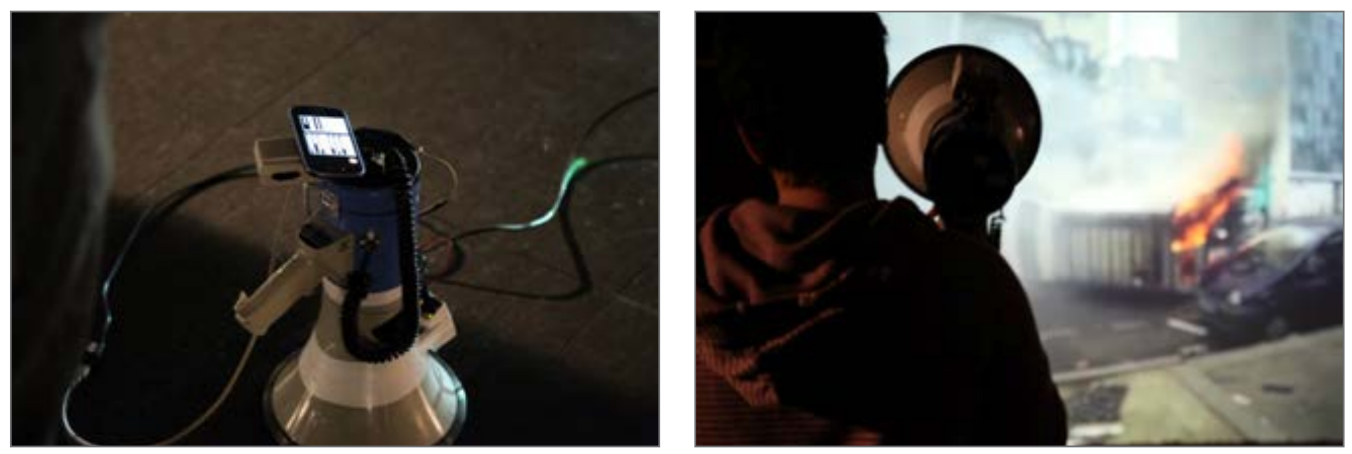

La interfaz gráfica de las pantallas de los MM se compone de los siguientes elementos: Dos apartados temáticos de protesta divididos en otras dos pantallas con sus respectivos botones para seleccionar la acción que se quiere hacer. El primer apartado (Pantalla 1) contiene los lemas que se pueden elegir y que se clasifican en tres tipos de contenido: lemas constructivos ( - 'No, no, no a la guerra', - 'Palestina libertad'), lemas destructivos (- 'Israel asesino del pueblo palestino'. - 'No es una guerra, es un genocidio') y lemas contra la policía, (- 'No, no, policía no'. - 'Perros guardianes del orden y la ley, asesinos armados, abuso de poder'). Los lemas constructivos suben el volumen de las noticias objetivas y bajan el de las noticias destructivas. Los destructivos, suben el volumen de las noticias destructivas y baja el de las noticias objetivas. Articular lemas contra la policía, acarrea la consecuencia de una carga policial, la consecuente subida de volumen de las noticias destructivas y la bajada de las noticias objetivas.

El segundo apartado es el que pertenece a la acción violenta. Estando en la pantalla 1, aparece un botón con la imagen de una llama en la zona superior 
derecha. Al pulsarlo, la interfaz pasa a la pantalla 2. Aquí, las opciones de violencia son tres: mobiliario, comercios y policía. Centrar la cólera manifestante en el mobiliario urbano tiene como consecuencia una carga policial. Destrozar comercios acarrea una respuesta superior al de la opción del mobiliario y violentar contra las fuerzas policiales implica el fin de la protesta a causa de la masiva intervención policial y sufrir la desacreditación absoluta del conjunto de los medios de comunicación.

Hay diferentes tipos de duelo. Un duelo se da cuando uno o varios MM realizan acción que intente cambiar el transcurso de la protesta. Los duelos suceden por secciones: por ejemplo, si MM1 elige luchar para sacar adelante la acción de romper mobiliario, en MM2 y MM3 se habilita la opción que permite luchar por evitar esa acción pulsando el botón 'evitar violencia'. En el momento de pulsar una elección, la interfaz del MM que ha sido pulsado se vuelve negra con una frase que instruye al usuario en la manera en que se debe de luchar: "Lucha por tu idea: Grita y agita el megáfono".

\subsection{DesCRIPCIÓN TÉCNICA}

Protesta- $\$ 1$ se compone de un ordenador MacBook Pro con sistema operativo OS X Lion 10.7.5 y tres MM. Cada uno de los MM contiene un megáfono, un smartphone con sistema operativo Android, un microcontrolador Arduino Uno y un acelerómetro DE-ACCM3D conectado al Arduino.

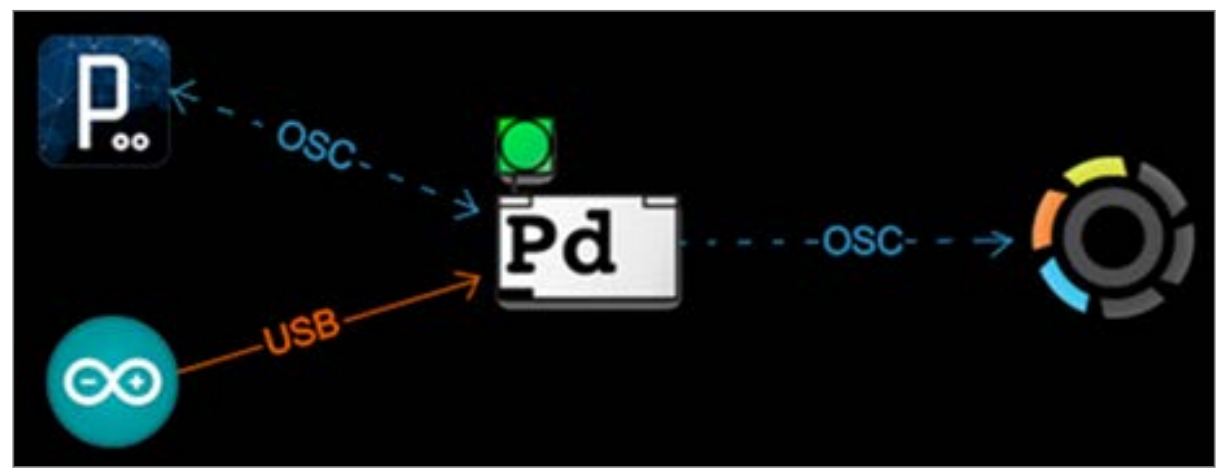

La aplicación de los smartphones está programado en el modo Android del software Processing. Los Arduinos llevan instalado el firmware 'Standard Firmata' y se controlan desde el ordenador (vía USB) con el programa Pure Data (en adelante PD) en su versión 'Extended' que incluye la librería 'Pduino'. El 
cerebro de la instalación es PD que controla todo desde el ordenador y recibe a través de los Arduinos los valores de los acelerómetros. Se comunica con Processing mediante el protocolo de comunicación OSC (Open Sound Control) quien usa el mismo protocolo para enviar mensajes a PD. El control de los vídeos lo hace el software Jitter (Max 6.1) que cumple las órdenes que PD le manda, también por OSC. Todos los mensajes OSC pasan por la red WIFI creada por un router al que se conectan los teléfonos móviles y el ordenador.

\section{CONCEPTO Y REFERENTES}

El 23 de septiembre de 2008, Google libera el código de Android para desarrolladores, haciendo posible la creación del HTC Dream: el primer teléfono inteligente con una plataforma open source basado en el sistema operativo Linux. Para entonces, ya existían lenguajes de código abierto como Pure Data (desde 1996) o Processing (desde 2001) que permitían programar música interactiva y obras multimedia apoyándose en microcontroladores u ordenadores. La revolución vino de la mano de la posibilidad de programar en un lenguaje abierto que se interpreta en un objeto cotidiano como es un smartphone.

Así, los artistas por primera vez tienen la oportunidad de crear proyectos para dispositivos inteligentes que contienen diversos sensores y que la gran mayoría de la sociedad usa diariamente. De esta manera, no solo se democratiza la accesibilidad al arte tecnológico sino que, además, existe la posibilidad de acceder a la experiencia artística simultáneamente desde diferentes lugares del mundo, de compartir la experiencia a través de internet en tiempo real o de difundir el proyecto desde el mismo objeto en un instante.

En paralelo, el año 2008, la red social Facebook supera el umbral de 100 millones de usuarios en todo el mundo. Este fenómeno supone un cambio en el modo de compartir información ya que, a partir de ese instante, 100 millones de personas pueden difundir, recibir e intercambiar información escrita y audiovisual en tiempo real casi desde cualquier lugar del planeta desde cualquier aparato con conexión a internet ${ }^{2}$. Actualmente, en el año 2016, Facebook cuenta ya con más de 1415 millones de usuarios, mientras otras redes sociales como Twitter (288 millones), Instagram (300 millones) o Whatsapp (700 millones) aumentan constantemente su número de usuarios ${ }^{3}$. Con estos medios, la información y el conocimiento se vuelve de fácil acceso y, en con- 
secuencia, el aprendizaje del desarrollo tecnológico se vuelve accesible para las personas no-especializadas.

Con todo, los artistas continúan sin poder construir muchos dispositivos tecnológicos debido al alto coste de las piezas y de la incapacidad de construir manualmente diversos accesorios con la precisión de una máquina.

En 2014, en pleno "boom" de las impresoras 3D (Tilves 2015) la empresa Arduino presenta su primera impresora 3D de diseño libre posibilitando a los creativos la construcción de cualquier objeto a partir de un modelo digital de tres dimensiones (Oyanedel 2014). Con esta democratización material, el panorama artístico se encuentra en un punto en el que la creación de casi cualquier objeto es posible.

Ese mismo año se produce la revolución de los drones (vehículos aéreos no tripulados) que permiten controlar y realizar acciones desde el aire, añadiendo una nueva posibilidad de intervención espacial al Arte (Ruibal 2014) y otorgan a las tecnologías ya existentes nuevas posibilidades de acción, como capturar planos aéreos en video con un bajo coste económico.

Paralelamente a la mencionada democratización tecnológica, varias protestas en todo el mundo comienzan a crecer y a hacerse oír mediante las redes sociales: La llamada Primavera Árabe del 2011 en los países árabes ${ }^{4}$, el Movimiento 15-M del 2011 en España ${ }^{5}$, Occupy Wall Street del 2011 en New York ${ }^{6}$ y los Indignados de la Plaza Sintagma del 2011 en Atenas (Sánchez-Vallejo, 2011), entre otros. Artistas como Ai Weiwei y Tania Bruguera, también usan las redes sociales para denunciar la opresión que sufren por parte de los gobiernos de China y Cuba, respectivamente, provocando la creación de campañas mundiales de apoyo a su causa. A su vez, muchos artistas comienzan a usar las tecnologías que antes no estaban a su alcance para unir el Arte con la acción, la creación con la calle y poner en tela de juicio la delgada línea que separa al Artivismo del Activismo.

En definitiva, la creación de proyectos de arte transmedia para protestar emerge con fuerza y comienza a reclamar su lugar como alternativa no-violenta de protesta: El colectivo No Somos Delito, crea en abril de 2015 la primera manifestación de hologramas en protesta de la Ley de Seguridad Ciudadana en Madrid7. Durante el Occupy Wall Street de 2011, Andy Noelker diseña "People's Skype" que permite a los asistentes mantener una multiconferencia ${ }^{8}$. Chris Csikszentmihalyi diseña en el año 2014 robots indignados con la inten- 
ción de enviarlos a manifestarse a Latinoamérica (Caballero 2014). En el 2015, Leo Selvaggio cede un modelo 3D de su rostro que permite engañar a los software de reconocimiento facial que usan las cámaras de vigilancia en Estados Unidos (Bejerano 2015)

Cabe mencionar a los videojuegos como disciplina artística ligada a la tecnología y a la protesta sociopolítica. Riot ${ }^{9}$ es un videojuego "simulador de Riot de 8 bits basado en hechos reales" (Pitcher 2013) cuyo atractivo se basa, precisamente, en que "supuestamente está basado en hechos reales" (Reeves 2013). La voluntad de simular, implica una actitud orientada a crear un entorno donde el usuario pueda sentirse parte del ficticio mundo creado y este videojuego ofrece la posibilidad de posicionarse en los disturbios en el bando de los manifestantes o en el de las fuerzas policiales aumentando el abanico de posibilidades de llevar a cabo acciones que determinen el transcurso de una manifestación.

Según la consultora Gartner Group, en 2020 habrá 26.000 millones de objetos conectados a la red debido al desarrollo del llamado "internet de las cosas" (Altares, 2015). Basándonos en la velocidad al que se desarrollan las tecnologías, en el abaratamiento de su coste y la socialización de su uso, es muy probable que el desarrollo de los proyectos artístico-tecnológicos evolucionen al mismo ritmo. Una vía de protesta pacífica y eficaz, que está demostrando su fuerza para ser un fuerte aliado de la voz de la lucha por el cambio.

\section{CONCLUSIONES Y NUEVAS VÍAS DE DESARROLLO}

Protest-\$1 permite aprender a protestar viviendo en tiempo real la simulación de las consecuencias que tendrían en una manifestación sociopolítica real las acciones que se llevan a cabo. Además interpela a los usuarios para ser conscientes de que en una protesta se pertenece a un grupo de personas unidas por una/s causa/s y que las acciones de una minoría pueden afectar a toda una multitud. Escuchar en tiempo real el modo en que los medios de comunicación adjetivan la protesta, habilita el aprender su forma de proceder para actuar en consecuencia: qué hacer y que no para que los medios transmitan una u otra opinión del evento de protesta. 
Al hilo de esto, se detecta un vacío en la falta de presencia de las redes sociales dada la capacidad de convocatoria y protesta que tienen, como se ha demostrado en varios casos mencionados anteriormente.

Por otro lado, la instalación limita mucho el abanico de opciones que el usuario puede elegir para protestar. Todo el rango de causas y consecuencias está limitado a una programación de código que no deja lugar a llevar a cabo más acciones de los que ofrece en los botones de la pantalla de los MM. Siendo consciente de la imposibilidad de crear un simulador que permita un número infinito de inputs, aquí se abre una vía de desarrollo del proyecto que permita una interacción menos controlada por el software y que de cabida a la capacidad de improvisación de los usuarios.

Finalmente, cabe destacar que la instalación une los conocimientos del arte y la tecnología orientados al cambio social e incentivando la participación cognitiva. A pesar de sus limitaciones, Protest- $\$ 1$ abre caminos para la implementación, investigación y desarrollo de proyectos de esta índole dirigiendo la creatividad y la innovación a la protesta sociopolítica.

\section{Referencias bibliográficas}

Altares, Guillermo. 2015. "Próxima frontera en Internet: La 'nube'". El País, 20 mayo. http://tecnologia.elpais.com/tecnologia/2015/05/15/actualidad/1431702172 096235.html

Bejerano, Pablo G. 2015. "Contra el reconocimiento facial: Entre la protesta y el arte". Eldiario.es, 21 abril. http://www.eldiario.es/turing/movimiento-anti-reconocimiento-facial_0_379712743.html

Caballero, Lucía. 2014. "Circuitos para la revolución: el artista que construye robots 'indignados'". Eldiario.es, 24 de noviembre. http://www.eldiario.es/hojaderouter/tecnologia/hardware/MIT-Media Lab-robots-tecnologia social-indignados 0 326167491.html

Durán, Luis. F. 2014. "Al menos 101 heridos, 67 de ellos policías y 29 detenidos en disturbios tras la 'marcha de la dignidad'”. El Mundo, 23 marzo. http://www.elmundo.es/madrid/2014/03/22/532de8c8268e3eeb178b4576.html

Giannetti, Claudia. 2002. Estética digital: Sintopía del arte, la ciencia y la tecnología. Barcelona: Associació de Cultura Contemporània l'Angelot

Martin, Abby. 2012. "Israel \& Palestine: S History of Occupation”. Emitido en Breaking the Set (RT America, Washington). Video de Youtube, 4:54. 16 noviembre. https://www.youtube. com/watch?v=IHnolK6juTs

Oyanedel, Juan Pablo. 2014. "Arduino presenta su primera impresora 3D de diseño libre". Fayerwayer.com, 1 octubre https://www.fayerwayer.com/2014/10/arduino-presenta-su-primera-impresora-3d-de-diseno-libre/ 
Pitcher, Jenna. 2013. "Riot, the 8bit riot simulator based on real life events". Polygon.com, 19 febrero. http://www.polygon.com/2013/2/19/4003884/riot-the-8bit-riot-simulator-based-onreal-life-events

Puente Montiel, Alexis. 2013. "La realidad fabricada o 'lo que no sale por la tele no existe'... guerras olvidadas, países olvidados". Miscelanea natural, 3 mayo. http://www.miscelaneanatural.org/derechos-y-libertades/la-realidad-fabricada-o-lo-que-no-sale-por-la-tele-no-existe-guerras-olvidadas-paises-olvidados

Reeves, Ben. 2013 "Fight the power with Riot". Game Informer Magazine, 23 febrero. http:// www.gameinformer.com/b/news/archive/2013/02/23/fight-the-power-with-riot.aspx/

Ruibal Pepe. 2014. "Drones, los nuevos artistas: Exposiciones de arte donde los autores son drones". Esquire, 19 diciembre. http://esquire.es/actualizacion/3607/drones-los-nuevos-ar$\underline{\text { tistas }}$

Sánchez-Vallejo María Antonia. 2011. "Los indignados griegos recuperan la plaza Sintagma". El País, 30 junio. http://internacional.elpais.com/internacional/2011/06/30/actualidad/1309384818 850215.html

Steyerl, Hito. 2002. "The articulation of protest". Republicart.net, september. Translated by Aileen Derieg. http://republicart.net/disc/mundial/steyerl02 es.pdf

Tilves, Mónica. 2015. ¿Sabes cuántas impresoras 3D se vendieron en 2014? Silicon, 2 abril. http://www.silicon.es/cuantas-impresoras-3d-se-vendieron-en-2014-78507

Tiqqun (Grupo). 2009. Llamamiento y otros fogonazos. Traducción, Ramón Vilatovà Pigrau y Alida Díaz Boadilla del Monte, Madrid: Acuarela. https://archive.org/details/Llamamiento

- 2013. "Teoría del Bloom". Tiqqunim (blog), enero. http://tiqqunim.blogspot.com.es/2013/01/ teoria-del-bloom.html

Tzu, Sun. (s. IV a.C.) 2013. El Arte de la Guerra. Barcelona: Medí

Notas

1 "22M, Dos millones de personas en una demostración de Dignidad", Marchas de la dignidad, 23 marzo 2014, entrada publicada por @edylat (activista en la PAH y RSP), http://marchasdeladignidad.org/22m-dos-millones-de-personas-en-una-demostracion-de-dignidad/

2 "Las redes sociales con más usuarios en activo en 2014". 2014. Go\&Web, 7 junio. http:// goandweb.com/cuantos-usuarios-hay-en-activo-en-las-redes-sociales-2014/

3 "Global social networks ranked by number of users 2016". 2016. Statista, The Statistics Portal. Acceso marzo de 2016, http://www.statista.com/statistics/272014/global-social-networksranked-by-number-of-users/

${ }^{4}$ Wikipedia, s.vv. "Primavera árabe" https://es.wikipedia.org/wiki/Primavera \%C3\%81rabe

${ }^{5}$ Movimiento15M, web oficial: http://www.movimiento15m.org/

${ }^{6}$ Occupy Wall Street, web oficial: http:/loccupywallst.org/

${ }^{7}$ Hologramasporlalibertad, web oficial: http://www.hologramasporlalibertad.org

${ }^{8}$ Peoples Sky: http://www.peoplesskype.org 
${ }^{9}$ Riot: Civil Unrest (gameplay), simulador de enfrentamientos entre manifestantes y policias dirigido por Leonard Menchiari, y desarrollado por los programadores Danilo Catalano, Claudio Mazza, Jendrik IIIner y Simon Michel. http://www.riotsimulator.org/. 